\title{
Damage evaluation and damage localization of rock
}

\author{
X.H. Xu ${ }^{\text {a }}$, S.P. Ma ${ }^{\text {b }}$, M.F. Xia ${ }^{\text {a }}$, F.J. Ke ${ }^{\text {a,c }}$, Y.L. Bai ${ }^{\text {a,* }}$ \\ a State Key Laboratory of Non-linear Mechanics (LNM), Institute of Mechanics, Chinese Academy of Sciences, \\ Beijing 100080, PR China \\ b Department of Geophysics, Peking University, Beijing 100871, PR China \\ c Department of Applied Physics, BeiHang University, Beijing 100083, PR China
}

\begin{abstract}
Knowledge of damage accumulation and corresponding failure evolution are prerequisite for effective maintenance of civil engineering so as to avoid disaster. Based on statistical mesoscopic damage mechanics, it was revealed that there are three stages in the process of deformation, damage and failure of multiscale heterogeneous elastic-brittle medium. These are uniformly distributed damage, localized damage and catastrophic failure. In order to identify the transitions from scattering damage to macroscopically localized one, a condition for damage localization was given. The experiments of rock under uniaxial compression with the aid of observations of acoustic emission and speckle correlation do support the concept of localization. This provides a potential approach to properly evaluate damage accumulation in practice. In addition, it is found in the experiments that catastrophic failure displays critical sensitivity. This gives a helpful clue to the prediction of catastrophic failure.
\end{abstract}

(C) 2004 Elsevier Ltd. All rights reserved.

Keywords: Damage localization; Critical sensitivity

\section{Introduction}

It is well known that rock undergoes damage evolution and failure under external loading. Then, properly evaluating damage accumulation and foreseeing failure are two of the major tasks of rock mechanics. Efforts have been made to study the process from damage evolution to even-

\footnotetext{
* Corresponding author.

E-mail address: baiyl@Inm.imech.ac.cn (Y.L. Bai).
}

tual failure [1-5]. It was found that there are three stages in the process of deformation, damage and failure of rocks. These are uniformly distributed damage, localized damage and catastrophic failure. Based on statistical mesoscopic damage mechanics [5,6], a transition condition from random damage to damage localization was derived and the result shows that damage localization can serve as an early precursor to failure.

This paper evaluates the damage evolution to failure of a heterogeneous elastic-brittle model. 
For comparison, with the aid of white-light digital speckle correlation method (DSCM) and acoustic emission (AE) technique, we observed the process of a gabbro under uniaxial compression. The observations demonstrate strain localization. In particular, the predicted damage localization transition point is very close to that of the experimental observation. Moreover, the experimental results also show that the rock might become sensitive to external loading prior to catastrophic failure, i.e. critical sensitivity $[7,8]$. This gives an experimental validation to the concept of critical sensitivity.

\section{Brief review of mean field (MF) approximation}

For the study of damage and failure of rock, a statistical model of heterogeneous elastic-brittle medium with mean field approximation was used $[7,8]$. It is assumed that such a sample consists of a number of linear elastic but brittle units, namely all units have the same elastic modulus $E_{0}$ but different breaking stress threshold $\sigma_{\mathrm{c}}^{*}$. Under monotonic loading, each unit remains elastic till its own $\sigma_{\mathrm{c}}^{*}$,

$\sigma_{\mathrm{s}}^{*}=\varepsilon_{\mathrm{s}}^{*} E_{0}$,

where $\sigma_{\mathrm{s}}^{*}$ and $\varepsilon_{\mathrm{s}}^{*}$ are mesoscopic stress and strain of each unit respectively. However, as soon as strain $\varepsilon_{\mathrm{s}}^{*}$ exceeds its mesoscopic threshold $\varepsilon_{\mathrm{c}}^{*}=\sigma_{\mathrm{c}}^{*} / E_{0}$ the unit breaks, namely the stress $\sigma_{\mathrm{s}}^{*}$ will drop from $\sigma_{\mathrm{c}}^{*}$ to zero. Suppose that the mesoscopic strength of units follows a probability distribution function $h\left(\sigma_{\mathrm{c}}^{*}\right)$, like Weibull distribution [9-11],

$h\left(\sigma_{\mathrm{c}}^{*}\right)=\frac{m}{\eta}\left(\frac{\sigma_{\mathrm{c}}^{*}}{\eta}\right)^{m-1} \mathrm{e}^{-\left(\frac{\sigma_{\mathrm{c}}^{*}}{\eta}\right)^{m}}$

where $m$ is the shape factor (Weibull modulus) and $\eta$ is the position factor of Weibull distribution respectively. The smaller the Weibull modulus $m$ is, the more diverse the threshold $\sigma_{\mathrm{c}}^{*}$ is, that is to say, the more heterogeneous the rock is. After taking dimensionless stress $\sigma=\sigma^{*} / \eta$ and normalized strain $\varepsilon=E_{0} \varepsilon^{*} / \eta$ (later, symbols for stress and strain without $*$ represent normalized stress and strain), Eq. (1) becomes $\sigma_{\mathrm{s}}=\varepsilon_{\mathrm{s}}$,

and Eq. (2) can be rewritten as

$h\left(\sigma_{\mathrm{c}}\right)=h\left(\varepsilon_{\mathrm{c}}\right)=m \sigma_{\mathrm{c}}^{m-1} \mathrm{e}^{-\sigma_{\mathrm{c}}^{m}}$.

The mean strength will be

$\bar{\sigma}_{\mathrm{c}}=\int_{0}^{\infty} \sigma_{\mathrm{c}} h\left(\sigma_{\mathrm{c}}\right) \mathrm{d} \sigma_{\mathrm{c}}=\Gamma(1+1 / m)$.

Under uniaxial monotonic loading, mean field approximation gives damage fraction $D$ as

$D=\int_{0}^{\sigma_{\mathrm{s}}} h\left(\sigma_{\mathrm{c}}\right) \mathrm{d} \sigma_{\mathrm{c}}=\int_{0}^{\varepsilon_{\mathrm{s}}} h\left(\varepsilon_{\mathrm{c}}\right) \mathrm{d} \varepsilon_{\mathrm{c}}$,

where $\sigma_{\mathrm{s}}$ and $\varepsilon_{\mathrm{s}}$ are the true dimensionless stress and strain of the sample respectively, in accord with mean field approximation. According to damage mechanics, the relation between the modulus $E$ of the damaged sample and the mesoscopic units' elastic modulus $E_{0}$ is

$E=E_{0}(1-D)$,

and the relations between the nominal and true variables, i.e. stress, strain and damage, are

$\sigma=\sigma_{\mathrm{s}}(1-D)$,

$\varepsilon=\varepsilon_{\mathrm{s}}$,

where $\sigma$ and $\varepsilon$ are nominal stress and strain respectively. Then, the constitutive relations, i.e. the relations between damage, nominal strain and stress, of the damaged sample with Weibull distribution (Eq. (2-a)) are

$\sigma=\varepsilon \mathrm{e}^{-\varepsilon^{m}}$,

$\sigma=(1-D)[-\ln (1-D)]^{\frac{1}{m}}$,

as well as,

$\varepsilon=[-\ln (1-D)]^{\frac{1}{m}}$.

\subsection{Macroscopic maximum stress}

From the constitutive relation (8) based on Weibull distribution, there is a maximum nominal stress point determined by the zero-slope condition of the constitutive curve. It is easy to obtain the expressions of the maximum nominal stress and 
the corresponding nominal strain and damage fraction as follows

$$
\sigma_{\max }=(e m)^{-\frac{1}{m}}, \quad \varepsilon_{\max }=m^{-\frac{1}{m}} \quad \text { and } \quad D_{\max }=1-\mathrm{e}^{-\frac{1}{m}} .
$$

\subsection{Criterion for damage localization}

It is important to predict the transition from uniformly distributed damage to damage localization. Physically, damage localization implies the emergence of macroscopic inhomogeneity, i.e. a prelude to localized rupture. The inhomogeneity of damage can be defined by

$$
\left(\frac{\partial D}{\partial x}\right) / D
$$

where $x$ is spatial coordinate. It is assumed that damage localization occurs once the rate of damage inhomogeneity starts to become positive [5,6], namely,

$$
\frac{\partial}{\partial t}\left[\left(\frac{\partial D}{\partial x}\right) / D\right] \geqslant 0
$$

where $t$ is time. Since $D$ is a function of nominal stress $D=D(\sigma)$, as expressed by Eq. (9), under the condition of constant gradient of nominal stress, the critical condition for damage localization was derived from Eq. (13) as [6]

$$
\begin{aligned}
& m \ln (1-D)[m(1-D) \ln (1-D)+1-2 D] \\
& \quad+(m-1) D=0 .
\end{aligned}
$$

The solution $D_{\mathrm{L}}$ to Eq. (14) denotes the critical damage for damage localization, provided the sample follows the above Weibull distribution. Then, the corresponding nominal stress $\sigma_{\mathrm{L}}$ and strain $\varepsilon_{\mathrm{L}}$ can be calculated from Eqs. (9) and (10) respectively.

For comparison of samples with various $m$, we denote relative nominal stress

$\underline{\sigma}=\sigma / \bar{\sigma}_{\mathrm{c}}$.

Then, the relative nominal stresses, characterizing damage localization and maximum nominal stress, are

$$
\underline{\sigma}_{\mathrm{L}}=\sigma_{\mathrm{L}} / \bar{\sigma}_{\mathrm{c}}
$$

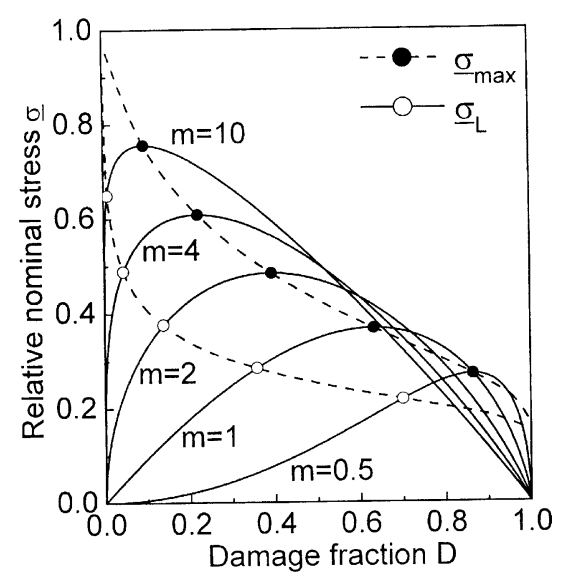

Fig. 1. The curves of relative nominal stress $\underline{\sigma}$ versus damage fraction $D$ for various Weibull $m$ (solid line -) (Eqs. (9) and (15)). Hollow circles (O) and the corresponding curve show the threshold of damage localization (Eqs. (9), (14) and (16)) and solid circles ( ) show the maxium nominal stress (Eqs. (11) and (17)) for various Weibull moduli $m$, respectively.

and

$\underline{\sigma}_{\max }=\sigma_{\max } / \bar{\sigma}_{c}$,

respectively.

The critical damage $D_{\mathrm{L}}$ and relative nominal stress $\underline{\sigma}_{\mathrm{L}}$ at damage localization, and the damage $D_{\max }$ and relative nominal stress $\underline{\sigma}_{\max }$ at maximum nominal stress for various Weibull moduli $m$ were calculated (see Fig. 1). It can be seen that $D_{\mathrm{L}}$ and $D_{\max }$ increase with decreasing Weibull modulus, while $\underline{\sigma}_{\mathrm{L}}$ and $\underline{\sigma}_{\max }$ have an opposite trend. That is to say, the more heterogeneous (less Weibull modulus $m$ ) the medium is, the more likely to rupture it is. Moreover, it is very clear that damage localization always appears ahead of maximum nominal stress under mean field approximation. So, damage localization may be one of the key factors to weaken the load-supporting capacity of a heterogeneous medium.

\section{Experimental observations of localization and critical sensitivity to failure}

\subsection{Experimental method}

Tests consist of rectangular gabbro samples, $5 \times 5 \times 13 \mathrm{~mm}^{3}$, that were loaded uniaxially with 


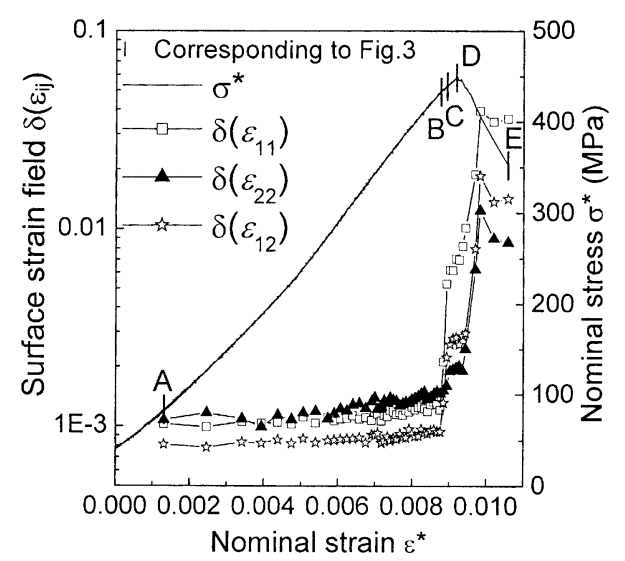

Fig. 2. Fluctuations of surface strain field $\delta\left(\varepsilon_{i j}\right)$ versus nominal strain $\varepsilon^{*}(\square, \boldsymbol{\Delta}$ and $\succsim)$ and experimental nominal stress-strain curve $\sigma^{*}-\varepsilon^{*}$ (solid line -), where $\sigma^{*}$ and $\varepsilon^{*}$ are experimental nominal stress and strain along loading axis. Moreover, points $\mathrm{A}, \mathrm{B}, \mathrm{C}, \mathrm{D}$ and $\mathrm{E}$ on the $\sigma^{*}-\varepsilon^{*}$ curve represent the initial stage of loading (A), experimental localization point (B), beyond localization (C), maximum nominal stress (D) and catastrophic failure $(\mathrm{E})$, respectively.

a MTS810 testing machine. The loading mode is boundary-displacement control with velocity of $0.02 \mathrm{~mm} / \mathrm{min}$. The displacement was measured by an extensometer with resolution of $3 \mu \mathrm{m}$ and an offset of load $1 \mathrm{kN}$. A typical nominal stress-strain curve is shown in Fig. 2 (solid line).

The surface of specimen was illuminated by a white luminescence and the speckle images were captured and transferred to computer by a CCD camera. After experiment, the speckle images were analyzed with digital speckle correlation method $[12,13]$, then, both displacement and strain fields during the loading process were obtained. Fig. 3 shows five stages (indicated in Fig. 2) of the deformation evolution of a gabbro sample.

On the other hand, two AE sensors were fixed on two sides of a sample with a specially designed clamp. The resonant frequencies of the sensors are $140 \mathrm{kHz}$ and $250 \mathrm{kHz}$ respectively. The AE signals were recorded and processed by an AE21-C system produced by the Institute of Computer Technology of Shenyang. As well known, AE is an effective method to detect damage process of rock, so the $\mathrm{AE}$ series, such as AE energy, can provide statistical information on damage evolution.

\subsection{Localization in rock experiments}

Based on the surface strain field, strain fluctuations can be measured by standard deviation of strain

$\delta\left(\varepsilon_{i j}\right)=\sqrt{\frac{1}{N} \sum_{k=1}^{N}\left[\left(\varepsilon_{i j}\right)_{k}-\bar{\varepsilon}_{i j}\right]^{2}} \quad(i, j=1,2)$,

where $\varepsilon_{i j}$ is strain tensor ( $i$ or $j=2$ indicates loading direction whereas 1 the direction vertical to loading (Fig. 3)), $\bar{\varepsilon}_{i j}$ is the mean value of the strain field, and $N=52531$ is the total sampling of the strain field. Fig. 2 shows the strain fluctuations $\delta\left(\varepsilon_{i j}\right)$ versus nominal strain $\varepsilon$. Clearly, in the range between A and B (Fig. 2), $\delta\left(\varepsilon_{i j}\right)$ has very low level (about 0.001 ) and the corresponding strain field remains nearly homogeneous (between A and B in Fig. 3). This indicates that the mean field approximation is valid before $\mathrm{B}$. Beyond $\mathrm{B}, \delta\left(\varepsilon_{i j}\right)$ increases significantly up to 0.01 (Fig. 2). This signifies a heterogeneous strain field. Especially, from C, D and $\mathrm{E}$ in Fig. 3, it is seen that high strain gradient appears. In other words, stain localization appears. Actually, from the experimental measurement, the localization point $\mathrm{B}$ is with the following nominal stress and strain

$\sigma^{*}=433 \mathrm{MPa}$ and $\varepsilon^{*}=0.00876$.

Also, the experiments show that strain localization (B in Fig. 2) occurs prior to the maximum nominal stress (D in Fig. 2), like damage localization predicted by mean field approximation (Fig. 1). Therefore, it can be deduced that the observed strain localization may be closely related to damage localization.

\subsection{Comparison of observed localization and theo- retical prediction}

In order to use Eq. (14) to predict damage localization quantitatively, we apply the theoretical model introduced in Section 2 to the gabbro compressive tests. Firstly, the parameters, such as $m, \eta$ and $E_{0}$ involved in Eqs. (1) and (2), should be determined according to the experimental nominal stress-strain $\left(\sigma^{*}-\varepsilon^{*}\right)$ curve. The slope of $\sigma^{*}-\varepsilon^{*}$ curve, 


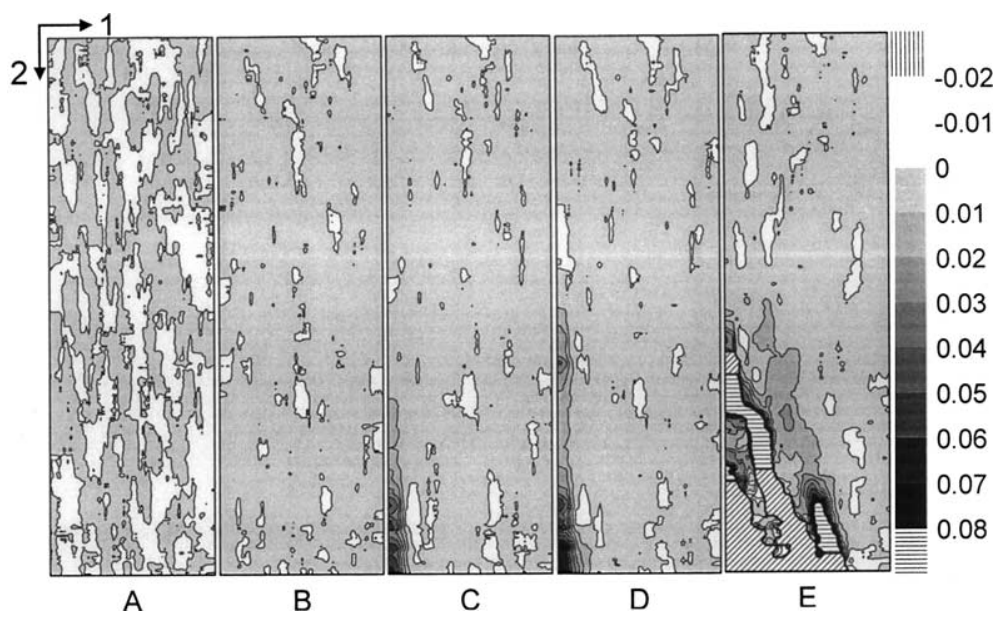

Fig. 3. Evolution patterns of surface strain field $\varepsilon_{11}$. The gabbro sample is compressed along axis 2. The five patterns A, B, C, D and E, corresponding to the points marked by the same letters in Fig. 2. indicates that the area where DSCM fails since the deformation is too big or the surface speckles fall off.

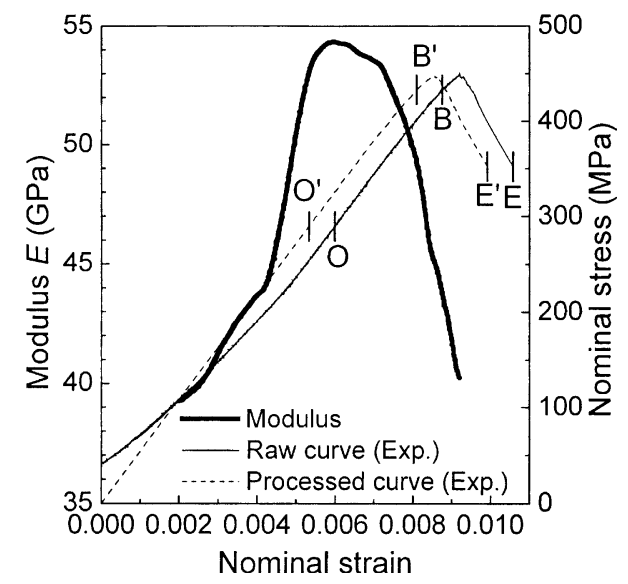

Fig. 4. Slope of $\sigma^{*}-\varepsilon^{*}$ curve, $\mathrm{d} \sigma^{*} / \mathrm{d} \varepsilon^{*}$ versus nominal strain $\varepsilon^{*}$ (bulk solid line -). The curves, $\sigma^{*}-\varepsilon^{*}$ (solid line -) and $\sigma^{* \mathrm{P}}-\varepsilon^{* \mathrm{P}}$ (dashed line - - -), are the raw experimental and processed experimental nominal stress-strain curves respectively . Points $\mathrm{O}$, $\mathrm{O}^{\prime}$ and $\mathrm{B}, \mathrm{B}^{\prime}$ are the points corresponding to maximum slope $\left(\mathrm{d} \sigma^{*} / \mathrm{d} \varepsilon^{*}\right)_{\mathrm{m}}$ and experimental localization, respectively.

$\frac{\mathrm{d} \sigma^{*}}{\mathrm{~d} \varepsilon^{*}}$

can be calculated (see Fig. 4). Clearly, the early increase in $\mathrm{d} \sigma^{*} / \mathrm{d} \varepsilon^{*}$ has no business with damage evolution. In addition, theoretically, Eqs. (1), (4) and (5) imply that $\mathrm{d} \sigma^{*} / \mathrm{d} \varepsilon^{*}$ decreases with increasing damage. So, we choose point $\mathrm{O}$ corresponding to maximum slope of $\sigma^{*} \varepsilon_{\varepsilon}^{*}$ curve, $\left(\mathrm{d} \sigma^{*} / \mathrm{d} \varepsilon^{*}\right)_{\mathrm{m}}$ as the starting point where the theoretical model can be used. On the other hand, as pointed out previously, the mean field approximation should be valid in the range between $\mathrm{O}$ and $\mathrm{B}$, and it is reasonable to assume that $\left(\mathrm{d} \sigma^{*} / \mathrm{d} \varepsilon^{*}\right)_{\mathrm{m}}$ is approximately equal to $E_{0}$ in Eq. (5). Hence, we can transform the raw experimental nominal stress-strain curve $\sigma^{*}-\varepsilon^{*}$ into its corresponding processed one $\sigma^{* \mathrm{P}}-\varepsilon^{* \mathrm{P}}$ by the following steps (Fig. 4): (1) Draw a straight line with slope $E_{0}$ from the origin $\left(\sigma^{* \mathrm{P}}=\varepsilon^{* \mathrm{P}}=0\right)$ to $\mathrm{O}^{\prime}\left(\mathrm{O}^{\prime}\right.$ and $\mathrm{O}$ with the same nominal stress). (2) Parallely shift the O-B-E-part of the raw stress-strain curve $\left(\sigma^{*}-\varepsilon^{*}\right)$ to point $\mathrm{O}^{\prime}$. Then, we obtain the whole processed experimental nominal stress-strain curve $\left(\sigma^{* \mathrm{P}}-\varepsilon^{* \mathrm{P}}\right.$, i.e. origin$\mathrm{O}^{\prime}-\mathrm{B}^{\prime}-\mathrm{E}^{\prime}$ in Fig. 4).

Now, start with the processed experimental $\sigma^{* \mathrm{P}}-\varepsilon^{* \mathrm{P}}$ to fit the undetermined parameters $E_{0}, m$ and $\eta$ in the model. Define a function

$f\left(E_{0}, \eta, m\right)=\sum_{i}\left(\sigma\left(\varepsilon_{i}^{\mathrm{P}}\right)-\sigma_{i}^{\mathrm{P}}\right)^{2}$,

where $\varepsilon_{i}^{\mathrm{P}}=E_{0} \varepsilon_{i}^{* \mathrm{P}} / \eta$ and $\sigma_{i}^{\mathrm{P}}=\sigma_{i}^{* \mathrm{P}} / \eta$ are normalized processed experimental nominal stress and strain respectively, and $\sigma\left(\varepsilon_{i}^{\mathrm{P}}\right)=\varepsilon_{i}^{\mathrm{P}} \mathrm{e}^{-\left(\varepsilon_{i}^{\mathrm{P}}\right)^{m}}$, (see Eq. (8)), is the theoretical nominal stress corresponding to $\varepsilon_{i}^{\mathrm{P}}$ with the three undetermined parameters $E_{0}, m$ and $\eta$. Using the data of $\sigma^{* \mathrm{P}_{-\varepsilon}}{ }^{* \mathrm{P}}$ in the range 


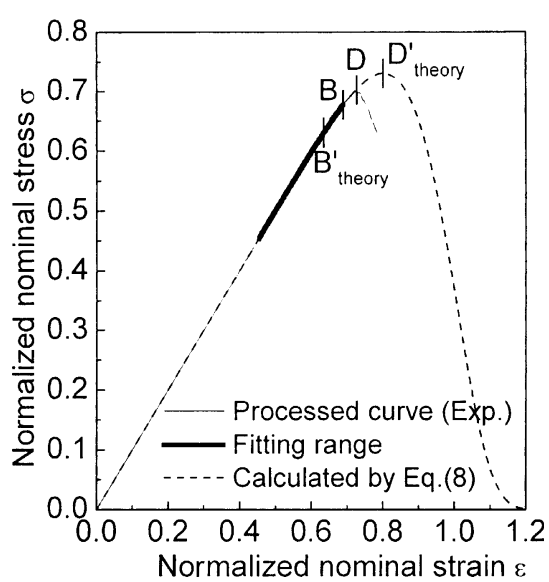

Fig. 5. Normalized nominal stress-strain curve $\sigma-\varepsilon$. Processed experimental nominal stress-strain curve (solid line -) and fitted nominal stress-strain curve (dashed line - - -). $\mathrm{B}^{\prime}$ and $\mathrm{B}_{\text {theory }}^{\prime}$ are the processed experimental and theoretical localisation points, respectively. $\mathrm{D}^{\prime}$ and $\mathrm{D}_{\text {theory }}^{\prime}$ are the processed experimental and theoretical maximum stress points, respectively.

between $\mathrm{O}^{\prime}$ and $\mathrm{B}^{\prime}$ to optimize function $f$, i.e. to minimize $f$,

$\min f\left(E_{0}, \eta, m\right)$.

Fit the three parameters $m, \eta$ and $E_{0}$ as

$m=10.7, E_{0}=54.4 \mathrm{GPa}$ and $\eta=640 \mathrm{MPa}$.

With these parameters, a theoretical nominal stress-strain curve can be calculated by Eq. (8) (see Fig. 5 (dashed line)). The agreement between the calculated and experimental nominal stressstrain curves are good prior to the peak D'. Moreover, according to Eqs. (9), (10) and (14), the theoretical damage localization occurs at $\mathrm{B}_{\text {theory }}^{\prime}$, and it is ahead of the experimental localization point B', i.e. expression (19) (see Fig. 5). The com- parisons of strains, stresses and damage for localization, calculated theoretically and measured experimentally are listed in Table 1.

Now, two conclusions can be drawn. The first is that damage localization may be closely related to strain localization. And the second is that theoretical condition for damage localization gives an early prediction of the occurrence of localization. Though localization implies a prelude to failure, it is by no means failure itself. In addition, from Fig. 5, it can be seen that the differences between the observed and predicted ones in peak load and failure are very distinct. This means that beyond localization the mean field approximation is no longer valid. So, in order to foresee failure more reasonably, we had to go further to find other precursors to failure. The promising candidate for this is critical sensitivity.

\subsection{Critical sensitivity in rock experiment}

Since the controlling variable is the boundary displacement $U$ in the experiment, a sensitivity of energy release to external boundary displacement $U$ is defined by

$S=\frac{U}{R} \frac{\Delta R}{\Delta U}$,

where $R$ is the response of acoustic emission energy $\Theta$ recorded by the acoustic emission system to boundary displacement $U$

$R=\frac{\Delta \Theta}{\Delta U}$.

The sensitivity in a gabbro test is shown in Fig. 6 . Note that the relation between the nominal stress and nominal strain presents a sudden failure beyond the maximum load. Correspondingly, the

Table 1

Comparison of experimental and calculated nominal strains and stresses of localization

\begin{tabular}{llllll}
\hline Localization point & $\varepsilon_{\mathrm{L}}^{*}\left(\varepsilon_{\mathrm{L}}^{* \mathrm{P}}\right)$ & $\sigma_{\mathrm{L}}^{*}\left(\sigma_{\mathrm{L}}^{* \mathrm{P}}\right)[\mathrm{MPa}]$ & $\varepsilon_{\mathrm{L}}\left(\varepsilon_{\mathrm{L}}^{\mathrm{P}}\right)$ & $\sigma_{\mathrm{L}}\left(\sigma_{\mathrm{L}}^{\mathrm{P}}\right)$ & $D_{\mathrm{L}}$ \\
\hline $\mathrm{B}$ & 0.00876 & 433 & 0.745 & 0.678 & - \\
$\mathrm{B}_{\text {theory }}$ & 0.00803 & 403 & 0.683 & 0.630 & 0.00764 \\
$\mathrm{~B}^{\prime}$ & 0.00810 & 433 & 0.689 & 0.678 & - \\
$\mathrm{B}_{\text {theory }}^{\prime}$ & 0.00747 & 403 & 0.635 & 0.630 & 0.00764 \\
\hline
\end{tabular}

$\mathrm{B}$ and $\mathrm{B}_{\text {theory }}$ are the raw experimental and calculated localization points respectively. $\mathbf{B}^{\prime}$ and $\mathrm{B}_{\text {theory }}^{\prime}$ are the processed experimental and calculated localization points respectively. 


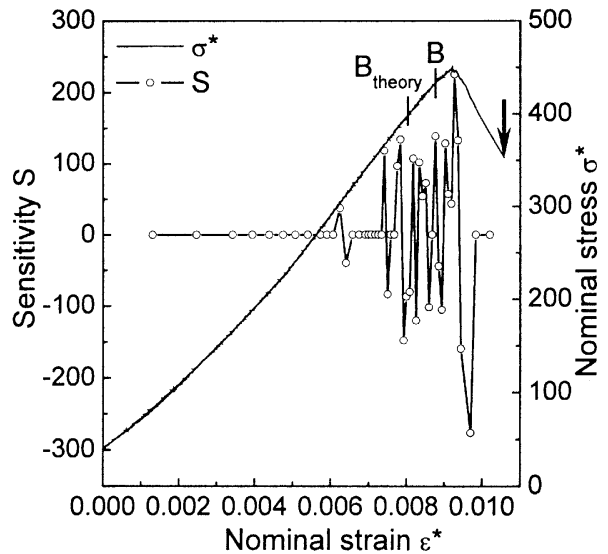

Fig. 6. Critical sensitivity in a rock experiment. The arrow indicates catastrophic failure.

sensitivity $S$ presents three different stages. At the first stage, the sensitivity remains low and nearly a constant. This is easy to understand. Then, it increases and oscillates. This roughly corresponds to the appearance of damage localization, as indicated by $\mathrm{B}$ and $\mathrm{B}_{\text {theory, }}$, the transition points of experimental and calculated damage localization respectively. Finally, the sensitivity increases very sharply ahead of eventual failure. Performed are 131 tests of gabbro. All tests show similar trend in critical sensitivity. So, it seems that critical sensitivity may provide a reasonable clue to failure.

\section{Discussions and conclusions}

1. At first stage of deformation, strain fluctuations in the rock test remain very low $\left(\sim 10^{-3}\right)$, mean field approximation seems to be valid there. And the agreement between theoretical and experimental nominal stress-strain curves is very good at this stage (Fig. 5). In addition, the predicted localization transition point based on mean field approximation is close to that from experimental observation.

2. Beyond localization, the fluctuations of strain field increase and MF approximation is no longer valid. it can be seen that the maximum nominal stress predicted by the MF approximation is greater than the experimental one (comparing $\mathrm{D}^{\prime}$ and $\mathrm{D}_{\text {theory }}^{\prime}$ in Fig. 5). In order to predict failure more closely, it is necessary to consider the effect of localization.

3. Experimental evidences show that critical sensitivity provides a precursor to catastrophic failure. This may provide a clue to the prediction of failure quite punctually.

4. Since the theoretical model neglects the elastic boundary condition acting on rock sample, it is unable to deal with rupture. Actually, as usual in experiments, the MTS tester is not rigid enough compared to the rock samples and its effect on failure must be considered. Such effects will be discussed in a later paper. In addition, the difference between rock samples and rock mass are significant and need further intensive study.

\section{Acknowledgments}

This work is supported by the National Natural Science Foundation of China (Grant No. 10172084, 10232050 and 10372012) and the Major State Research Project "Nonlinear Science" G200007735.

\section{References}

[1] J.W. Rudnicki, J.R. Rice, Conditions for the localization of deformation in pressure-sensitive dilatant materials, J. Mech. Phys. Solids 23 (1975) 371-394.

[2] Y. Berthaud, J.M. Torrenti, C. Fond, Analysis of localization in brittle materials through optical techniques, Exp. Mech. 37 (1997) 216-220.

[3] P. Bésuelle, J. Desrues, S. Raynaud, Experimental characterization of the localization phenomenon inside a Vosges sandstone in a triaxial cell, Int. J. Rock Mech. Min. Sci. 37 (2000) 1223-1237.

[4] X.P. Zhou, Q.L. Ha, Y.X. Zhang, K.S. Zhu, Analysis of formation localization and the complete stress-strain relation for brittle rock subjected to dynamic compressive loads, Int. J. Rock Mech. Min. Sci. 41 (2004) 311-319.

[5] Y.L. Bai, M.F. Xia, F.J. Ke, H.L. Li, Damage field equation and criterion for damage localization, in: R. Wang (Ed.), Rheology of Bodies with Defects, Kluwer Academic Publishers, Dordrecht, 1998, pp. 55-66.

[6] F.J. Ke, H.L. Li, Z.K. Jia, M.F. Xia, Y.L. Bai, Effect of heterogeneous on catastrophe rupture, in: A. Donnellan, P. Mora (Eds.), 3rd ACES Workshop Proceedings, GOPRINT, Brisbane, 2002, pp. 15-22. 
[7] Y.J. Wei, M.F. Xia, F.J. Ke, X.C. Yin, Y.L. Bai, Evolution induced catastrophe and its predictability, Pure Appl. Geophys. 157 (2000) 1945-1957.

[8] X.H. Zhang, X.H. Xu, H.Y. Wang, M.F. Xia, F.J. Ke, Y.L. Bai, Critical sensitivity in driven nonlinear threshold systems, Pure Appl. Geophys. 161 (2004) 19311944.

[9] W. Weibull, A statistical distribution function of wide applicability, J. Appl. Mech. ASME 18 (1951) 293-297.
[10] B.D. Coleman, On the strength of classical fibers and fiber bundles, J. Mech. Phys. Solids 7 (1958) 60-70.

[11] A.S. Jayatilaka, Fracture of Engineering Brittle Materials, Applied Sciences Publishers Ltd., London, 1979.

[12] W.H. Peter, W.F. Randson, Digital imaging techniques in experimental stress analysis, Opt. Eng. 21 (1981) 427-431.

[13] P. Zhou, K.E. Goodson, Subpixel displacement and deformation gradient measurement using digital image/speckle correlation (DISC), Opt. Eng. 40 (2001) 1613-1620. 